\title{
Appropriateness of knee MRI prescriptions in a hospital of Birjand, 2014
}

\section{Marjan Vejdani ${ }^{1}$, Yasamin Molavi Taleghani ${ }^{2}$, Amir Reza Kachooei ${ }^{3}$, Roghayeh Hosseini ${ }^{4}$, Sirvan Salehi ${ }^{4}$, Samira Foji ${ }^{5}$, Maryam Goudarzian ${ }^{1}$, Ghasem Fattahzadeh-Ardalani ${ }^{6} *$}

\author{
${ }^{1}$ Iranian Research Center on Healthy Aging, Sabzevar University of Medical Sciences, Sabzevar, Iran \\ ${ }^{2}$ PHD student of Health Services Administration, Health Management and Economics Research Center, School of \\ Management and Medical Information Sciences, Isfahan University of Medical Sciences, Isfahan, Iran \\ ${ }^{3}$ Department of Orthopedics, Ghaem Hospital, Mashhad University of Medical Sciences, Mashhad, Iran \\ ${ }^{4}$ Department of MRI, Vali-e-Asr Hospital, Birjand University of Medical Sciences, Birjand, Iran \\ ${ }^{5}$ School of Paramedical, Sabzevar University of Medical Sciences, Sabzevar, Iran. \\ ${ }^{6}$ Department of Neurology, School of Medicine, Ardabil University of Medical Sciences, Ardabil, Iran
}

Received: 10 November 2015

Accepted: 04 December 2015

\section{*Correspondence:}

Dr. Gasem Fattahzadeh-Ardalani,

E-mail: g.fattahzadeh2015@gmail.com

Copyright: (c) the author(s), publisher and licensee Medip Academy. This is an open-access article distributed under the terms of the Creative Commons Attribution Non-Commercial License, which permits unrestricted non-commercial use, distribution, and reproduction in any medium, provided the original work is properly cited.

\section{ABSTRACT}

Background: Knee pain is one of the most common complains which cause patients to visit a doctor and Magnetic Resonance Imaging (MRI) is one of the common diagnostic methods which are common to find the cause of Knee pain. This study aim to determine knee MRI appropriateness, in Valiasr hospital of Birjand in 2014.

Methods: In this study descriptive - analytical study, 150 patients referred to MRI unit were selected using convenient sampling method. Data were gatherers using a Questionnaire and then analyzed by SPSS16 using descriptive statistics and Pearson, chi-square and Fisher's exact tests.

Results: Of 150 cases, $70(46.7 \%)$ were considered inappropriate, 7 (4.7\%) were uncertain, and $73(48.7 \%)$ were deemed to be appropriate. The economic burden of inappropriate prescriptions was 46200000 Rails. A statistical significant relation was observed between appropriateness of prescription with sex, jobs, education, physician specialist and referring institution.

Conclusions: Considering the high rate of inappropriate prescriptions, it is necessary to find the causes. Developing, customization, and perform clinical guidelines in all of our country may help reducing this rate.

Keywords: Appropriateness, MRI, Hospital

\section{INTRODUCTION}

There are various methods of medical imaging, using specific waves or radiation. One of these methods is MRI. ${ }^{1}$ The use of MRI is increasing because of its noninvasiveness and precision compared with other clinical diagnostic techniques, ${ }^{2}$ and because it avoids using iodinated contrast agents and does not need recovery time. ${ }^{3}$ However, it is expensive and time-consuming and not all patients have access to it. Long waiting time for MRI patients is another limitation of the technology. ${ }^{4}$

The knee joint is the most important joint which tolerates the body weight and is under mechanical pressure in all activities such as standing, walking and even sitting. This pressure is due to carrying objects or just the body weight 
even if the person is in a fixed position. Various disorders are common within and around the knee joint. The level of joint injuries is high in developing countries, including Iran, due to progress in their use of vehicles and development of various sports without adequate preventive measures. Such injuries can cause acute problems and even lead to disability. Therefore, correct diagnosis of these injuries before invasive intervention will be very helpful in improving the final result. ${ }^{5}$

Inappropriate and unnecessary care is an economic issue related to quality of service, payment systems, financing of services, induced demands, etc. Iran, like other developing countries, suffers from the problem of inadequate resources. With such lack of resources, it is expected that available resources be used efficiently and effectively and technologies be utilized that reduce waste of resources. MRI prescription in the country is higher than the international standard and a lot of unnecessary MRIs are prescribed. For unknown reasons, this diagnostic service is prescribed more than it is needed. ${ }^{6}$

In a study conducted by Lehnert and Bree, $74 \%$ of CT and MRI examinations were reported as appropriate and $26 \%$ as not appropriate. Inappropriate examinations included MRI of lumbar spine, knee and shoulder. ${ }^{7}$ Ebrahimipour and et al studied the appropriateness of knee MRI examinations in Mashhad and found that $54.8 \%$ were inappropriate, $14.8 \%$ were uncertain and $30.4 \%$ were appropriate. The cost of inappropriate prescriptions was 38159100 IRR $^{8}{ }^{8}$ Therefore, there have been concerns regarding inappropriate prescription of MRI.

Experts from the Ministry of Health have proposed developing clinical guidelines as a way to control induced services, so that physicians can prescribe based on such guidelines. To prevent wasting patients' time, reducing costs incurred by patients and medical services, and giving priority to those really in need, it is necessary that medical services be provided in accordance with clinical guidelines. ${ }^{9}$

Since unnecessary MRI prescriptions impose substantial financial burden on insurers and patients ${ }^{8}$ and the knee joint is of high priority areas for MRI examinations ${ }^{7}$, this study aimed to "determine the appropriateness of knee MRI prescriptions in Vali-e-Asr Hospital of Birjand in 2014".

\section{METHODS}

In this descriptive-analytical study, the population included all patients referring to MRI unit of Vali-e-Asr hospital in Birjand for knee MRI examination. During the first 6 months of 2014, 150 of them were selected by random sampling. Inclusion criteria included patients with knee MRI prescription and exclusion criteria were patients with a history of knee surgery. The estimated number of subjects was 150 considering $\mathrm{P}=0.26$ and $\mathrm{d}=$
0.07 as in the study by Lehnert and Bree. ${ }^{7}$ Data were collected via field research and a questionnaire administered to the patients. The questionnaire consisted of 12 questions related to demographic characteristics and questions related to MRI prescription. The questionnaire validity and reliability were determined by Salari et al. ${ }^{10}$ The Clinical Guidelines (CGs) developed by Ebrahimipour et $\mathrm{al}^{8}$ was used to identify prescription appropriateness. The patients' clinical histories were examined by experienced specialists who were involved in the Clinical Guidelines development, so that the prescription appropriateness can be determined in accordance with the guide. Finally, with regard to the level of costs incurred by patients and insurers for knee MRI, the financial burden imposed on them was calculated. Data were analyzed using SPSS16, via descriptive statistics and Pearson, and Chi-square tests and Fisher's exact tests. Level of significance was set at $\mathrm{p}<0.05$.

\section{RESULTS}

Of 150 patients participated in the study, $(\mathrm{N}=110 ; 73.3 \%)$ were male. Most patients $(\mathrm{N}=61 ; 40.7 \%)$ were $20-30$ years old and most of them $(\mathrm{N}=61 ; 40.7 \%)$ had college education. In terms of occupation, 48 patients $(32.0 \%)$ were employed; $149(99.3 \%)$ had insurance and 30 $(20.0 \%)$ had complementary insurance; $143(95.3 \%)$ were examined by a physician. In 148 cases $(98.7 \%)$ MRI had been prescribed by a specialist. The referral institution was private offices for 110 patients $(73.3 \%)$ and public hospitals for 40 patients $(26.7 \%)$. 119 MRI requests $(79.3 \%)$ were made by an orthopedist, 1 request $(0.7 \%)$ by a rheumatologist and 30 requests $(20.0 \%)$ by other specialties. Frequency of knee MRI examinations by appropriateness is given in Table 1. The Chi-Square test showed significant differences between the prescription appropriateness and the patients' gender and occupation; the specialty of the prescriber; and the referral institute. Moreover, Spearman test showed a significant relationship between the administration and education on patients. $(\mathrm{P}<0.05)$

Table 1: Frequency of Knee MRI prescription by appropriateness.

\begin{tabular}{|ll|}
\hline Appropriateness & Frequency/percentage \\
\hline Appropriate & $73(48.7 \%)$ \\
\hline Uncertain & $7(4.7 \%)$ \\
\hline Inappropriate & $70(46.7 \%)$ \\
\hline Total & $150(100 \%)$ \\
\hline
\end{tabular}

The knee MRI cost was 660,000 IRR in 2014. Social security and Medicare insurance paid $70 \%$ of the amount and the remaining $30 \%$ is payable as deductible by the patient. The Armed Forces Insurance paid $90 \%$ of the costs with the patient paying a $10 \%$ share. Other insurance companies including those for banks, and oil companies paid $100 \%$ of the costs. The results showed that of the total financial burden resulting from 
inappropriate prescribing, $70 \%$ was incurred by insurance companies. In Table 2, the financial burden imposed on patients and insurance is presented.

Table 2: Estimated costs of inappropriate knee MRI prescribing among patients.

\begin{tabular}{|lllll|}
\hline Insurance type & $\begin{array}{l}\text { Inappropriate } \\
\text { prescribing }\end{array}$ & $\begin{array}{l}\text { Costs incurred by } \\
\text { patients (IRR, \%) }\end{array}$ & $\begin{array}{l}\text { Costs incurred by } \\
\text { insurers (IRR, \%) }\end{array}$ & $\begin{array}{l}\text { Total burden } \\
\text { (IRR, \%) }\end{array}$ \\
\hline Health care & $18(5.7 \%)$ & $3564000(30 \%)$ & $8316000(70 \%)$ & 11880000 \\
\hline Social Security & $35(50 \%)$ & $6930000(30 \%)$ & $16170000(70 \%)$ & 23100000 \\
\hline Armed Forces & $11(15.7 \%)$ & $726000(10 \%)$ & $6534000(90)$ & 7260000 \\
\hline Banks & $2(2.8 \%)$ & 0 & $1320000(100)$ & 1320000 \\
\hline Uninsured & $4(5.7 \%)$ & $2,640,000(100 \%)$ & 0 & 2640000 \\
\hline Total & $70(100)$ & $13860000(30 \%)$ & $32340000(70 \%)$ & 46200000 \\
\hline
\end{tabular}

\section{DISCUSSION}

The study showed that $70 \%$ of the financial burden was incurred by the insurance companies. If half of the country's MRI examinations is considered inappropriate (regardless of uncertain prescriptions), at least half the charges paid by the insurers as reimbursement or by patients as deductible for MRI is in fact the financial burden imposed intentionally or unintentionally by doctors on patients and insurers. The increasing costs of health care worldwide have led health economics experts and practitioners in all countries to seek new ways to control costs. ${ }^{11}$

Charles E.P in a study showed that the increasing but non-effective health care costs stating that based on different studies, inappropriate and unnecessary care was about $30-15 \%$ reaching more than $40 \%$ in some therapies. According to him, the US spent about $\$ 100$ million in unnecessary medical treatments which is a very high figure. ${ }^{12}$ Cutler and et al in a study showed that an average one-third of conventional medical tests and procedures performed for patients is unnecessary or uncertain. $^{13}$

Inappropriate and unnecessary care is an economic issue related to quality of service, payment systems, financing of services, induced demands, etc. Iran, like other developing countries, suffers from the problem of inadequate resources. Despite the lack of resources, it is expected that available resources be used efficiently and effectively and technologies be utilized that would not waste resources. MRI prescription in the country is higher than the international standard and a lot of unnecessary MRIs are prescribed. For unknown reasons, this diagnostic service is prescribed more than it is needed. ${ }^{6}$

Salari et al study to determine clinical appropriateness of lumbar spine MRI prescriptions showed that among 300 lumbar spine MRI examinations, 167 were inappropriate. They calculated the costs of inappropriate prescriptions to be 88009000 IRR. $^{10}$ Ebrahimipour and et al studied the appropriateness of knee MRI examinations in Mashhad and found that $54.8 \%$ were inappropriate, $14.8 \%$ were uncertain and $30.4 \%$ were appropriate with the cost of 38159100 IRR. $^{8}$ The huge financial burden imposed on insurance companies and patients shows the importance of using such evidence by policy makers.

In the current study, a statistically significant relationship was observed between prescription appropriateness and the referral institute, so that the number of inappropriate prescriptions in patients admitted to clinics was significantly higher than those admitted to hospitals. The results of a study conducted about inappropriate lumbar MRI prescriptions in Shiraz showed that a smaller percentage of patients referred from public hospitals with inappropriate prescription. It seems that physicians in hospitals spend more time examining patients. ${ }^{14}$ However, in Ebrahimipour and et al in a study on inappropriate knee MRI prescriptions in Mashhad wasn't found a significant relationship between these variables. ${ }^{8}$

The results of this study showed a significant association between inappropriate prescriptions and the patients' gender, with inappropriate prescriptions being higher among men than among women. This is consistent with the results by Keshtakaran et al. ${ }^{14}$ However, no significant association between inappropriate prescriptions and the patients' gender was found in the study conducted in Mashhad. ${ }^{8}$

According to results of the present study, no significant relationship was observed between inappropriate prescriptions and the patients' occupation. Self-employed subjects had more inappropriate prescriptions. The results of the study carried out in Shiraz showed that students and housewives had the highest percentage of inappropriate prescriptions. ${ }^{14}$ However, Ebrahimpour and et al found no significant difference between inappropriate prescriptions and the patients' occupation. ${ }^{8}$

Limitations of this study include: distribution of knee MRI examinations during the day, long duration of data 
collection period and lack of cooperation on the part of some patients.

\section{CONCLUSION}

The results of this study showed that the rate of inappropriate knee MRI prescriptions was more than expected. Thus the use of Clinical Guidelines can be a step towards standardization of treatment and prevention of additional costs.

\section{ACKNOWLEDGEMENTS}

Authors would like to thank all the participants who consented to participate in the study. Authors also thank those students of nursing sociology who helped in collecting data.

\section{Funding: No funding sources}

Conflict of interest: None declared

Ethical approval: Not required

\section{REFERENCES}

1. Yazdani S, Mirshahi S. A case of displacement metallic foreign object intraocular during MRI. Journal of Ophthalmology Bina 2002;8(2):186-90.

2. Rezaei Y, Rahimnia A, Mirmohamad SM, Vaziri K, Fakhrejahani F. Sensitivity and specificity of MRI and Arthroscopy in knee joint injuries. Tehran University Medical Journal 2007;65(9):47-52.

3. Ryahi ÂN, Salar S, Rahimi S. Designing protocol of MR for angiography of lower limbs using MRI instrument 1.5 Tasla . J Mazandaran Univ Med Sci 2004;14(42):98-107.

4. Barzin M, Abdi R, Gholmohamadi H. Diagnostic accuracy of ultrasonography in comparison with magnetic resonance imaging in patients with knee trauma. J J Gorgan Uni Med Sci 2012;14(3):92-7.

5. Ebrahimipour H, Mirfeizi SZ, Vafaee Najar A, Kachooei AR, Ariamanesh ASH, Ganji R, et al. Developing an Appropriateness Criteria for Knee MRI Using the Rand Appropriateness Method (RAM)-2013. Arch Bone Jt Surg 2014;2(1):47-51.
6. Ostovar R, Rashidian A, Pourreza A, Rashidi BH, Hantooshzadeh S, Ardebili HE, et al. Developing criteria for cesarean section using the RAND appropriateness method. BMC Pregnancy Childbirth 2010;10:52.

7. Lehnert BE, Bree RL. Analysis of appropriateness of outpatient CT and MRI referred from primary care clinics at an academic medical center: how critical is the need for improved decision support? Journal of the American College of Radiology: JACR 2010;7(3):192-7.

8. Ebrahimipour H, Mirfeizi SZ, Vejdani M, Vafaeenajar A, Kachooei A, Ariamanesh AS, et al. Evaluation of Medical costs resulting from Magnetic Resonance Imaging inappropriate prescriptions for Knee joint, using RAND Method in Ghaem Hospital- 2013. Hakim Research Journal 2015;17(4).

9. News IR. Administration unnecessary medical services and treatment, way to earn more income. 201380651732.

10. Salari H, Ostovar R, Esfandiari A, Keshtkaran A, Sari AA, Manesh HY, et al. Evidence for Policy Making: Clinical Appropriateness Study of Lumbar Spine MRI Prescriptions Using RAND Appropriateness Method. International Journal of Health Policy and Management 2013;1:1-5.

11. Bergh TH, Steen K, Lindau T, Soldal LA, Bernardshaw SV, Lunde L, et al. Costs analysis and comparison of usefulness of acute MRI and 2 weeks of cast immobilization for clinically suspected scaphoid fractures. Acta Orthop 2015;86(3):303-9.

12. Charles EP. The methodologic fondations of studies of the appropriateness of medical care. The England Journal of Medicine 1993;1329(17):1241-45.

13. Cutler MD. A Guide to Health Care Reform. Journal of Economic Perspectives. 1994;8(3):13-29.

14. Keshtkaran A, Bagheri MH, Ostovar R, Salari H. Developing Criteria for Lumbar Spine Magnetic Resonance Imaging (MRI) using RAND Appropriateness Method (RAM). Iran J Radiol 2012;9(3).

Cite this article as: Vejdani M, Taleghani YM, Kachooei AR, Hosseini R, Salehi S, Foji S, et al. Appropriateness of knee MRI prescriptions in a hospital of Birjand, 2014. Int J Sci Rep 2015;1(8): 299-302. 